Test method

\title{
Characterization of the viscoelastic-plastic properties of UPVC by instrumented sharp indentation
}

\author{
Guangjian Peng ${ }^{\mathrm{a}}$, Yihui Feng ${ }^{\mathrm{b}}$, Yong Huan ${ }^{\mathrm{b}}$, Taihua Zhang ${ }^{\mathrm{a}, \mathrm{b}, *}$ \\ ${ }^{a}$ Key Laboratory of EEM (Zhejiang University of Technology), Ministry of Education E' Zhejiang Province, Hangzhou 310014, China \\ b State Key Laboratory of Nonlinear Mechanics (LNM), Institute of Mechanics, Chinese Academy of Sciences, Beijing 100190, China
}

\section{A R T I C L E I N F O}

\section{Article history:}

Received 17 July 2013

Accepted 30 August 2013

Keywords:

Viscoelasticity

Creep compliance

Yield strength

Instrumented indentation

UPVC

\begin{abstract}
A B S T R A C T
The yielding behavior of glassy polymers may be sensitive to the hydrostatic pressure and also be influenced by the strain rate. Thus, the yield strength is a function of hydrostatic pressure and strain rate instead of a constant. In this paper, using dimensional analysis and finite element simulations, we established a method to estimate the yield strength of glassy polymers by instrumented indentation. Together with the method for determining shear creep compliance proposed in our previous work, the viscoelastic-plastic properties of glassy polymers can be extracted from a single indentation load-depth curve. Applications were illustrated on unplasticized polyvinyl chloride (UPVC). The shear creep compliance and yield strength of UPVC were successfully determined by a single indentation test. Using these parameters, the true stress-strain curves of UPVC under different strain rates were approximately predicted according to a linear viscoelastic-perfectly plastic constitutive description.
\end{abstract}

(c) 2013 Published by Elsevier Ltd.

\section{Introduction}

Instrumented indentation is an efficient and convenient tool for probing mechanical properties of small volumes of material and small structures. The methods for determining the elastic [1] and plastic [2-6] properties of elastic-plastic solids by instrumented indentation have been well established. For viscoelastic-plastic materials such as glassy polymers, the deformations are complicated. Not only time-independent elastic-plastic deformation, but time-dependent viscoelastic deformation occurs during indention tests. Thus, the characterization of the viscoelastic-plastic properties of glassy polymers by instrumented indentation face challenges. To reduce the difficulty, researchers usually isolate the elastic, viscoelastic

\footnotetext{
* Corresponding author. Postal address: LNM, No. 15 Beisihuanxi Road, Beijing 100190, China. Tel.: +86-10-82543950.

E-mail address: zhangth@lnm.imech.ac.cn (T. Zhang).
}

and plastic deformations and determine the corresponding material parameters separately.

\subsection{Determination of elastic modulus}

For elastic-plastic solids, the widely used method is to extract elastic modulus from the initial unloading stiffness (also called contact stiffness), $S=(d F / d h)_{\mathrm{u}}$, of the unloading curve [1]. It is based on Sneddon's work [7], which relates $S$, the reduced modulus, $E_{\mathrm{r}}$, and the projected contact area, $A$,

$E_{r}=\frac{\sqrt{\pi}}{2 \beta} \frac{S}{\sqrt{A}}$

where $\beta$ is a correction factor for indenter shape $(\beta=1.034$ for a Berkovich indenter and $\beta=1.012$ for a Vickers indenter $[8,9])$. Taking into account the effect of a non-rigid indenter, the reduced modulus, $E_{\mathrm{r}}$, is defined as

$$
\frac{1}{E_{r}}=\frac{1-\nu^{2}}{E}+\frac{1-\nu_{i}^{2}}{E_{i}}
$$


where $E, \nu$ and $E_{\mathrm{i}}, \nu_{\mathrm{i}}$ are the elastic modulus and Poisson's ratio of sample and indenter, respectively. For a diamond indenter, $E_{\mathrm{i}}=1141 \mathrm{GPa}$ and $\nu_{\mathrm{i}}=0.07$. Thus, if Poisson's ratio of the sample is known, the elastic modulus of the sample can be determined by Eq. (2), when the reduced modulus is obtained by Eq. (1).

The projected contact area, $A$, in Eq. (1) can be determined from the contact depth, $h_{\mathrm{c}}$. For a perfect Berkovich indenter, the projected contact area is calculated by $A=24.5 h_{c}^{2}$. Oliver and Pharr [1] put forward a formula for calculating contact depth

$h_{c}=h_{u}-\varepsilon \frac{F_{u}}{S}$

where $h_{\mathrm{u}}$ and $F_{\mathrm{u}}$ are the depth and load at the beginning of unloading, respectively; $\varepsilon$ is a constant related to indenter shape ( $\varepsilon=0.72$ for sharp indenters).

For viscoelastic-plastic solids, however, the deformation is time-dependent and more complex. Due to the effect of creep, a platform appears during holding, and the unloading part of the load-depth curve is more convex [dotted curve in Fig. 1(b)] than that for elastic-plastic materials. This leads to overestimating the contact stiffness. If the
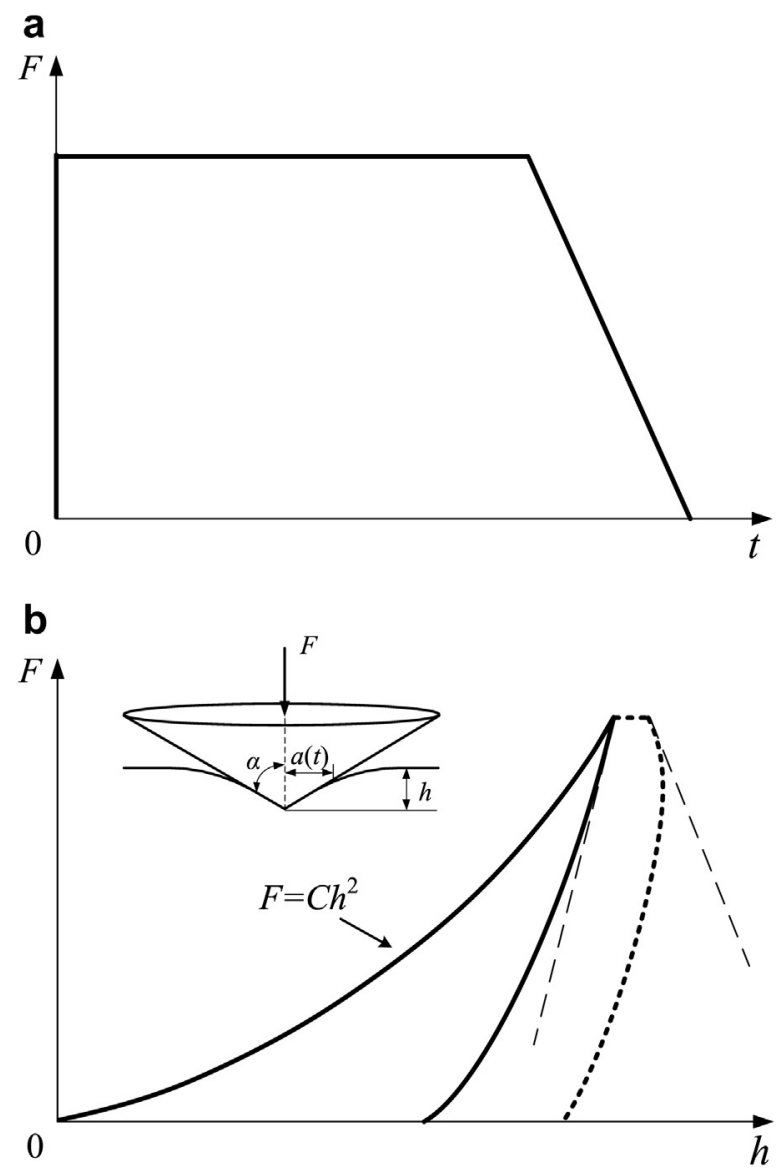

Fig. 1. Schematic illustrations of (a) step-hold-unload loading profile and (b) load-depth curves of indentation tests (dotted curve is the typical holding and unloading curve of viscoelastic solids). unloading rate is sufficiently low, a nose may be evident and the contact stiffness is negative. Using the measured contact stiffness, $S$, to determine the elastic modulus could introduce considerable error. In this case, the widely used Oliver-Pharr method [1] is inapplicable for extracting elastic modulus [10-14]. Feng et al. [10] have proposed that the true (elastic) contact stiffness, $S_{\mathrm{e}}$, can be calculated from the measured contact stiffness, $S$, using the following equation

$\frac{1}{S_{e}}=\frac{1}{S}+\frac{\dot{h}_{h}}{\left|\dot{F}_{u}\right|}$

where $\dot{h}_{\mathrm{h}}$ is the creep rate $(d h / d t)$ at the end of holding, $\dot{F}_{\mathrm{u}}$ is the unloading rate $(d F / d t)$ at the beginning of unloading. Tang et al. [12] proposed a revised Oliver-Pharr method by replacing $S$ in Eqs. (1) and (3) with $S_{\text {e }}$. Using the revised Oliver-Pharr method, the contact depth and the reduced modulus can be accurately determined.

\subsection{Determination of shear creep compliance}

In order to characterize the time-dependent behavior, the creep compliance should be determined. Lee and Radok [15], Hunter [16], Graham [17] and Ting [18] have developed theories for the problem of indentation in linear viscoelastic solids, which give the load-depth $(F-h)$ relationship

$h^{(n+1) / n}(t)=\frac{1-\nu}{4 C_{n}} \int_{0}^{t} J(t-\tau) \frac{d F(\tau)}{d \tau} d \tau$

where $J(t)$ is the shear creep compliance; $C_{n}$ is a constant related to indenter shape, $n=1, C_{1}=\tan \alpha / \pi$ for a sharp indenter, and $\alpha$ is the included half-angle; $n=2, C_{2}=2 \sqrt{ } R / 3$ for a spherical indenter, and $R$ is the radius of a spherical indenter. Based on this relationship, several methods for determining shear creep compliance have been proposed [19-26]. A step-hold-unload loading profile [Fig. 1(a)] is often used to determine the shear creep compliance, and the corresponding formula is $[19,24,25]$

$J(t)=\frac{4 C_{n}}{(1-\nu) F_{0}} h^{(n+1) / n}(t)$

where $F_{0}$ is the maximum load for holding. It should be noted that these methods require the deformation of materials in the linear viscoelastic regime. In practice, it is difficult to meet this requirement for glassy polymers such as polymethylmethacrylate (PMMA) and unplasticized polyvinyl chloride (UPVC), because the high stresses beneath the indenter tip can easily lead to these materials yielding. Lu et al. [19] and Tweedie et al. [22] pointed out that the response can be regarded as approximately linear viscoelastic when the indentation is shallow enough for plastic deformation to be negligible. However, it is difficult to identify the critical indentation load or depth that does not cause significant plastic deformation.

Aimed at avoiding these difficulties, Peng et al. [25] put forward a convenient method for determining the shear creep compliance of linear viscoelastic-plastic solids when 
significant plastic deformation occurs during the indention test. A step-hold-unload loading profile [Fig. 1(a)] and a Berkovich indenter were adopted in their tests. They firstly revised the $F$ - $h$ curve by removing the plastic deformation from the measured $F$ - $h$ curve through a three-step procedure, and then used the revised $F$ - $h$ curve to determine the shear creep compliance. The revised depth $h_{\mathrm{re}}(t)$ can be obtained by

$$
h_{r e}(t)= \begin{cases}\sqrt{\pi F(t) /\left(2 \gamma E_{r} \tan \alpha\right)} & \text { for loading } \\ h(t)-h_{0}^{p}, & \text { for holding - unloading }\end{cases}
$$

where $F(t)$ is the load for loading segment; $\gamma$ is a correction factor; $h_{0}^{\mathrm{p}}$ is the maximum plastic depth contained in loading and unloading segments. Replacing the measured depth, $h(t)$, in Eq. (6) with the revised depth, $h_{\mathrm{re}}(t)$, the shear creep compliance of linear viscoelastic-plastic solids can then be accurately determined.

\subsection{Determination of plastic properties}

In order to characterize the plastic behavior by instrumented indentation, a constitutive description and an accurate method for extracting the material parameters in the constitutive description from the $F$ - $h$ curve are necessary. To date, a number of methods [2-6] have been proposed for determining the plastic properties of elastic power-law hardening materials such as pure and alloyed engineering metals. For these materials, two material parameters - the yield strength, $\sigma_{\mathrm{y}}$, and the strain hardening exponent, $n$, - need to be determined. One of the most complete studies was presented by Dao et al. [2]. They used dimensional analysis to construct a set of dimensionless functions to characterize instrumented sharp indentation. With the aid of finite element (FE) simulations, they derived several analytical expressions that relate indentation data to plastic properties from these dimensionless functions. The yield strength and strain hardening exponent can then be extracted from the $F$ - $h$ curve by using these analytical expressions. For glassy polymers, however,

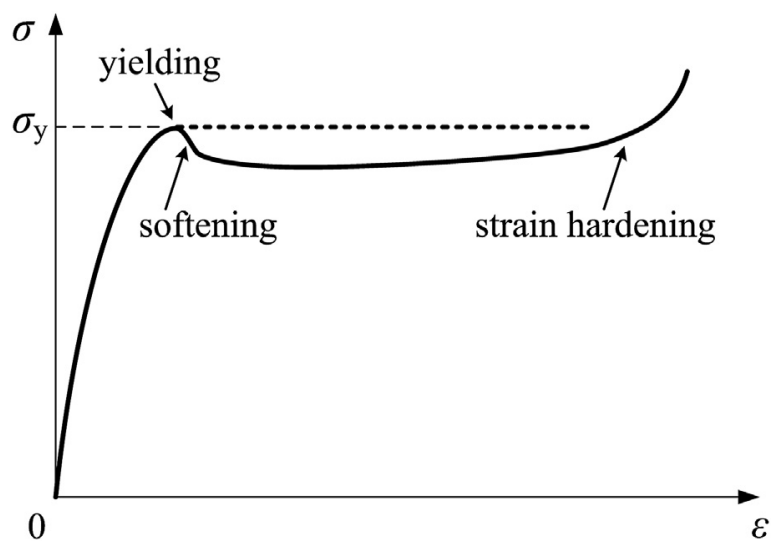

Fig. 2. Schematic illustrations of the typical constitutive description of glassy polymers (solid curve), and a viscoelastic-perfectly plastic description (dotted curve). the elastic power-law hardening description is inappropriate. The typical constitutive description of glassy polymers (solid curve in Fig. 2) may include viscoelastic deformation before yielding, softening and strain hardening after yielding [27]. Furthermore, the yielding behavior of glassy polymers may be sensitive to the hydrostatic pressure and also be influenced by the strain rate [28-30]. As a consequence, these methods based on elastic power-law hardening description are inapplicable for glassy polymers.

To reduce the complexity of the plastic constitutive model, the behavior of glassy polymers can be approximated by a linear viscoelastic-perfectly plastic description (dotted curve in Fig. 2). The yield strength of glassy polymers is a function of hydrostatic pressure and strain rate, which can be expressed as [31-33]

$\sigma_{y}(P, \dot{\bar{\varepsilon}})=\sigma_{y 0}+k P+B \ln (\dot{\bar{\varepsilon}})$

where $\sigma_{\mathrm{y} 0}$ is the yield strength under the pure shear deformation; $k$ is the coefficient of internal friction, which reflects the effect of hydrostatic pressure on the yield strength; $P=-\sigma_{k k} / 3$ is the hydrostatic pressure; $B$ is the coefficient that reflects the effect of strain rate on the yield strength; $\dot{\bar{\varepsilon}}=\sqrt{(1 / 2) \dot{\varepsilon}_{i j} \dot{\varepsilon}_{i j}}$ is the effective strain rate. There are three material parameters, i.e. $\sigma_{\mathrm{y} 0}, k$ and $B$, in the constitutive description that need to be determined to characterize plastic behavior. Considering the effect of hydrostatic pressure and ignoring the effect of strain rate on yield strength, Zhang et al. [33] put forward a method for determining $\sigma_{\mathrm{y} 0}$ and $k$ by instrumented indentation using two sharp indenters. However, the accuracy is somewhat unsatisfactory. As addressed in their paper [33], the yield strength of PMMA determined by indentation test is $108 \mathrm{MPa}$, and the uniaxial tensile result is $85 \mathrm{MPa}$. The relative error is as high as $27 \%$. Thus, a more accurate method to determine plastic properties of glassy polymers is needed.

In the present work, we consider a sharp indenter (conical, Berkovich or Vickers) indenting linear viscoelastic-plastic solids with a step-hold-unload loading profile [Fig. 1(a)]. It is assumed that elastic-plastic deformation is dominant and viscoelastic deformation can be neglected during the fast loading segment, and there is only viscoelastic deformation during the holding segment. Therefore, viscoelastic and plastic properties can be determined separately from different segments of the $F-h$ curve. Using dimensional analysis and FE simulations, a new method for extracting the yield strength under the pure shear deformation, $\sigma_{\mathrm{y} 0}$, and the coefficient of internal friction, $k$, is established. When $\sigma_{\mathrm{y} 0}$ and $k$ are determined, together with the shear creep compliance, $J(t)$, determined in our previous work [25], the viscoelastic-plastic properties of glassy polymers can be characterized.

\section{A new method for determination of plastic properties}

If glassy polymers are regarded as approximate linear viscoelastic-perfectly plastic materials, there are three material parameters, i.e. $\sigma_{\mathrm{y} 0}, k$ and $B$, in Eq. (8), that need to be determined in order to characterize the plastic 
properties. For the reason that the relationship between effective strain rate, $\dot{\bar{\varepsilon}}$, and indentation strain rate, $\dot{\varepsilon}_{I}=\dot{h} / h$, is unknown, it is not possible to know how the effective strain rate affects yield strength. The coefficient $B$ that reflects the effect of strain rate on the yield strength cannot be determined by instrumented indentation. Thus, in the present work, the coefficient $B$ that can be determined by uniaxial tensile tests at different strain rates is assumed to be known, and we merely consider the method for determining the yield strength under the pure shear deformation, $\sigma_{\mathrm{y} 0}$, and the coefficient of internal friction, $k$, by instrumented indentation. The dimensional analysis and FE simulations are used to construct expressions that relate the indentation data to elastic-plastic properties.

\subsection{Dimensional analysis}

To extract $\sigma_{\mathrm{y} 0}, k$ and shear creep compliance $J(t)$ from a single $F$ - $h$ curve, we considered a sharp indenter indenting linear viscoelastic-perfectly plastic solids with a step-holdunload loading profile [Fig. 1(a)], which is the same as that used in our previous work [25]. It is assumed that (1) elastic-plastic deformation is dominant and viscoelastic deformation is negligible during the fast loading; (2) in the holding segment, only viscoelastic process occurs. Thus, during loading, the load, $F$, is regarded as irrelevant to the viscoelastic properties, and it can be written as

$F=f_{1}\left(h ; E, \nu, \sigma_{y 0}, k ; E_{i}, \nu_{i}, \alpha\right)$

where $E, \nu$ and $E_{\mathrm{i}}, \nu_{\mathrm{i}}$ are the elastic modulus and Poisson's ratio of sample and indenter, respectively; $\alpha$ is the included half-angle of a sharp indenter. When $\alpha$ is fixed, Eq. (9) can be rewritten as

$F=f_{2}\left(h ; E, \nu, \sigma_{y 0}, k ; E_{i}, \nu_{i}\right)$

Eq. (10) is often simplified by combining the elasticity effects of the indenter and sample as

$F=f_{3}\left(h ; E_{r}, \sigma_{y 0}, k\right)$

where $E_{\mathrm{r}}$ is the reduced modulus as defined in Eq. (2). Applying the $\Pi$ theorem [34] in dimensional analysis, Eq. (11) becomes

$$
\frac{F}{E_{r} h^{2}}=\Pi_{1}^{A}\left(\frac{\sigma_{y 0}}{E_{r}}, k\right)
$$

where $\Pi_{1}^{A}$ is a dimensionless function.

Based on the above assumption (1) that elastic-plastic deformation is dominant during loading, the response of sharp indentation during loading can be described by Kick's law [Fig. 1(b)]

$F=C h^{2}$

where $C$ is the loading curvature. Replacing $F / h^{2}$ with $C$ in Eq. (12) leads to

$$
\frac{C}{E_{r}}=\Pi_{1}^{A}\left(\frac{\sigma_{y 0}}{E_{r}}, k\right)
$$

Alternatively, Eq. (14) can be written as

$\frac{E_{r}}{C}=\Pi_{1}\left(\frac{E_{r}}{\sigma_{y 0}}, k\right)$

where $\Pi_{1}$ is a dimensionless function. It is obvious from Eq. (15) that, when the indenter shape and included half-angle are fixed, the loading curvature $C$ is independent of indentation depth, but depends on material properties.

During unloading, the unloading stiffness, $d F / d h$, is related to viscoelastic-plastic properties. For fixed indenter shape and included half-angle, the unloading stiffness is given by

$\frac{d F}{d h}=g_{1}\left(h, h_{u}, t ; E, \nu, \sigma_{y 0}, k, \eta ; E_{i}, \nu_{i}\right)$

where $h_{\mathrm{u}}$ is the depth at the beginning of unloading; $\eta$ is the viscosity coefficient of the sample that reflects the creep (viscous) properties, $t$ denotes time. Similarly, combining the elasticity effects of the indenter and sample, Eq. (16) can be rewritten as

$\frac{d F}{d h}=g_{2}\left(h, h_{u}, t ; E_{r}, \sigma_{y 0}, k, \eta\right)$

Using the $\Pi$ theorem, Eq. (17) becomes

$\frac{d F}{d h}=E_{r} h \Pi_{2}^{A}\left(\frac{h_{u}}{h} ; \frac{\sigma_{y 0}}{E_{r}}, k, \frac{\eta}{E_{r} t}\right)$

Evaluating Eq. (18) at $h=h_{\mathrm{u}}$ leads to

$S=\left.\frac{d F}{d h}\right|_{h=h_{u}}=E_{r} h_{u} \Pi_{2}^{B}\left(\frac{\sigma_{y 0}}{E_{r}}, k, \frac{\eta}{E_{r} t}\right)$

where $S$ is the contact stiffness. It is clear in Eq. (19) that the contact stiffness is relevant to both elastic-plastic properties and creep properties. As discussed in section 1.1, the effect of creep $\left(\eta / E_{\mathrm{r}} t\right)$ can be removed by replacing the measured contact stiffness, $S$, with the true (elastic) contact stiffness, $S_{\mathrm{e}}$. Then, Eq. (19) is written as

$\frac{S_{e}}{E_{r} h_{u}}=\Pi_{2}\left(\frac{E_{r}}{\sigma_{y 0}}, k\right)$

where $S_{\mathrm{e}}$ can be calculated using Eq. (4). From the two dimensionless functions, $\Pi_{1}$ and $\Pi_{2}$, and with the help of FE simulations, analytical expressions can be derived to relate the indentation data to the elastic-plastic properties.

\subsection{FE simulations}

We considered a conical indenter with an included halfangle of $70.3^{\circ}$ indenting an infinite half-space. Since it is an axisymmetric problem, an axisymmetric two-dimensional FE model (Fig. 3) was constructed in the commercial finite element program ABAQUS to simulate the indentation response of elastic-perfectly plastic solids. The size of the sample was set to ten times larger than the radius of the contact region, so that the sample can be regarded as an infinite half-space. The sample was modeled using fournode bilinear axisymmetric quadrilateral elements, and composed of a fine mesh of 2736 elements near the contact 


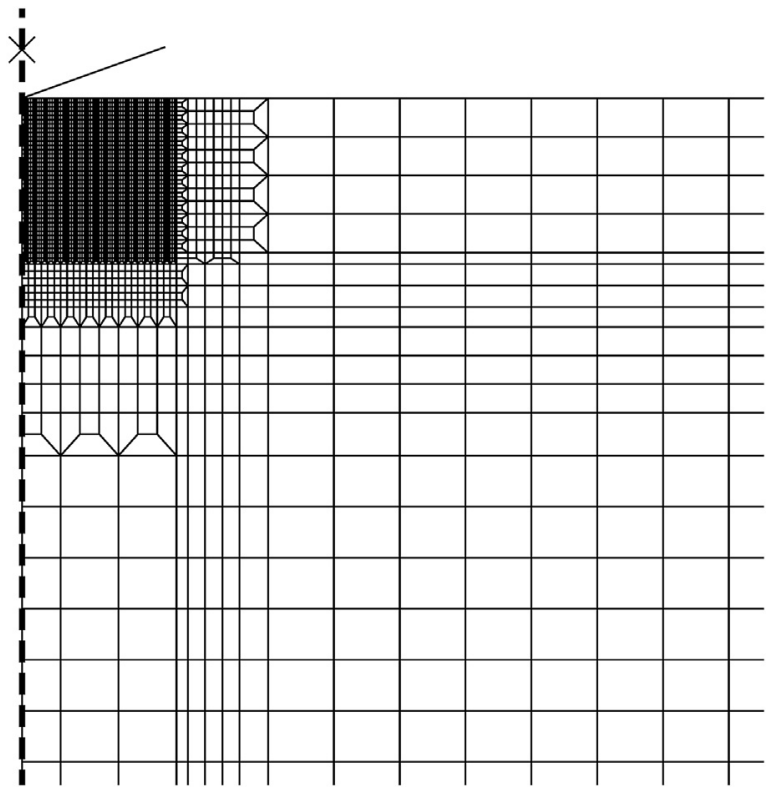

Fig. 3. Mesh design for axisymmetric FE simulations.

region and a gradually coarser mesh of 1031 elements further from the contact region. The indenter was modeled as a rigid body, and the contact was modeled as frictionless.

As analyzed in section 2.1, for glassy polymers, the effect of creep properties is negligible during fast loading, and can also be removed during unloading by replacing the measured contact stiffness with the true (elastic) contact stiffness. Thus, in the FE simulations, the sample was defined as an elastic-perfectly plastic material for simplification. To define an elastic-perfectly plastic material, four material parameters (the elastic modulus, $E$, the Poisson's ratio, $\nu$, the yield strength under the pure shear deformation, $\sigma_{\mathrm{y} 0}$, and the coefficient of internal friction, $k$ ) need to be input into ABAQUS. We considered a wide range of material parameters of glassy polymers. As listed in Table 1 , each material parameter takes 4 values, and the combination of these parameters leads to $4^{4}=256$ combination materials. Eliminating some impossible combinations (e.g. $E=0.1 \mathrm{GPa}$ and $\sigma_{\mathrm{y} 0}=130 \mathrm{MPa}$, where the yield strength is greater than the elastic modulus), conical indentations in 180 materials were simulated to construct the expressions that relate indentation data to the elastic-plastic properties.

\subsection{FE results}

The correlations between $E_{\mathrm{r}} / \sigma_{\mathrm{y} 0}$ and $E_{\mathrm{r}} / C$ are illustrated in Fig. 4. It clearly shows the influence of the coefficient of internal friction on the evolution of the dimensionless function $\Pi_{1}$ [Eq. (15)]. For each coefficient of internal friction, a linear relationship exists between $E_{\mathrm{r}} / \sigma_{\mathrm{y} 0}$ and $E_{\mathrm{r}} / C$. Fitting a linear equation, $E_{\mathrm{r}} / \sigma_{\mathrm{y} 0}=a\left(E_{\mathrm{r}} / C\right)+b$, to the data points of each coefficient of internal friction, a set of best-fit slopes and intercepts was obtained: $a=94.740$,
Table 1

Material parameters input into ABAQUS for defining materials.

\begin{tabular}{llcl}
\hline$E(\mathrm{GPa})$ & $\nu$ & $\sigma_{\mathrm{y} 0}(\mathrm{MPa})$ & $k$ \\
\hline 0.1 & 0.33 & 10 & 0 \\
2.5 & 0.38 & 50 & 0.10 \\
5.0 & 0.43 & 90 & 0.25 \\
10 & 0.48 & 130 & 0.40 \\
\hline
\end{tabular}

$b=-42.170$ for $k=0 ; a=116.11, b=-53.024$ for $k=0.10$; $a=162.50, b=-76.698$ for $k=0.25$ and $a=236.13$, $b=-114.54$ for $k=0.40$. We found that the slope, $a$, increases and the intercept, $b$, decreases linearly with the coefficient of internal friction (Fig. 5)

$a=274.06 k+92.476$

and

$b=-(139.67 k+41.003)$

Thus, the relationship between $E_{\mathrm{r}} / \sigma_{\mathrm{y} 0}$ and $E_{\mathrm{r}} / C$ can be described by the following expression

$\frac{E_{r}}{\sigma_{y 0}}=(274.06 k+92.476) \frac{E_{r}}{C}-(139.67 k+41.003)$

Fig. 6 shows the relationship between $S_{\mathrm{e}} / E_{\mathrm{r}} h_{\mathrm{u}}$ and $E_{\mathrm{r}} / \sigma_{\mathrm{y} 0}$. It can be seen that all data points lie approximately on a single curve, which means the relationship between $S_{\mathrm{e}} / E_{\mathrm{r}} h_{\mathrm{u}}$ and $E_{\mathrm{r}} / \sigma_{\mathrm{y} 0}$ is insensitive to the coefficient of internal friction. The evolution of the dimensionless function $\Pi_{2}$ [Eq. (20)] is relatively easy. Fitting a logarithmic equation, $S_{\mathrm{e}} /$ $E_{\mathrm{r}} h_{\mathrm{u}}=c \ln \left(E_{\mathrm{r}} / \sigma_{\mathrm{y} 0}\right)+d$, to the data points in Fig. 6, we obtained

$\frac{S_{e}}{E_{r} h_{u}}=0.95661 \ln \left(\frac{E_{r}}{\sigma_{y 0}}\right)+1.7181$

Using Eq. (23) and Eq. (24), the yield strength under pure shear deformation, $\sigma_{\mathrm{y} 0}$, and the coefficient of internal

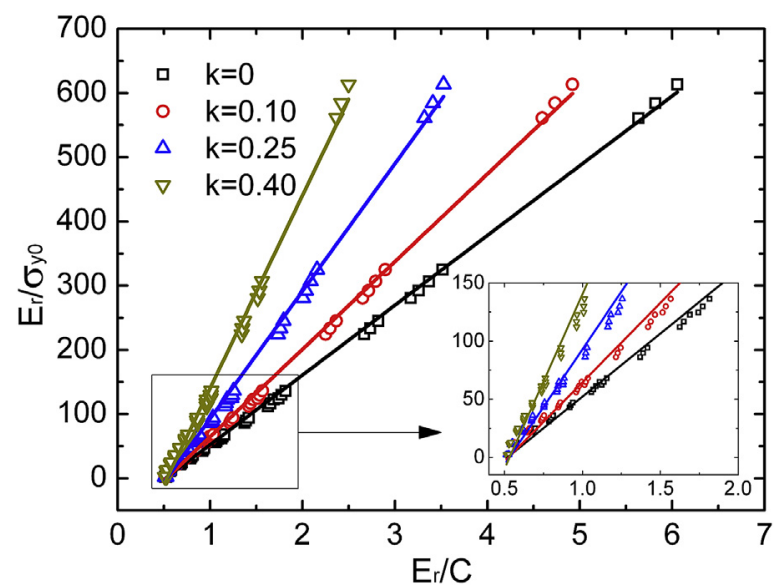

Fig. 4. Dimensionless function $\Pi_{1}$ constructed in the parametric study with 180 simulations. Solid lines are the best-fitting straight lines to the data points of different $k$. 

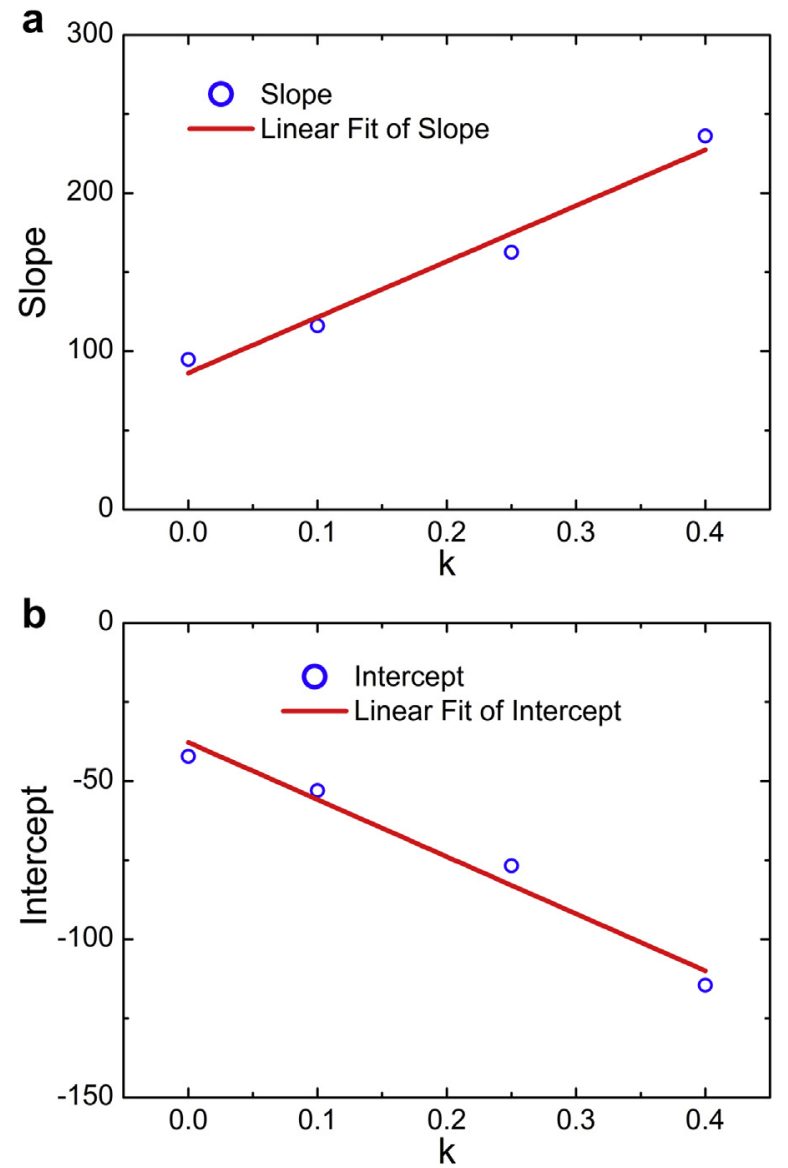

Fig. 5. Effects of the coefficient of internal friction on (a) the slope and (b) the intercept of fitting lines in Fig. 4.

friction, $k$, can be extracted from $F$ - $h$ curves if the reduced modulus, $E_{\mathrm{r}}$, is known, where the reduced modulus of glassy polymers can be determined by using the revised Oliver-Pharr method proposed by Tang et al. [12].

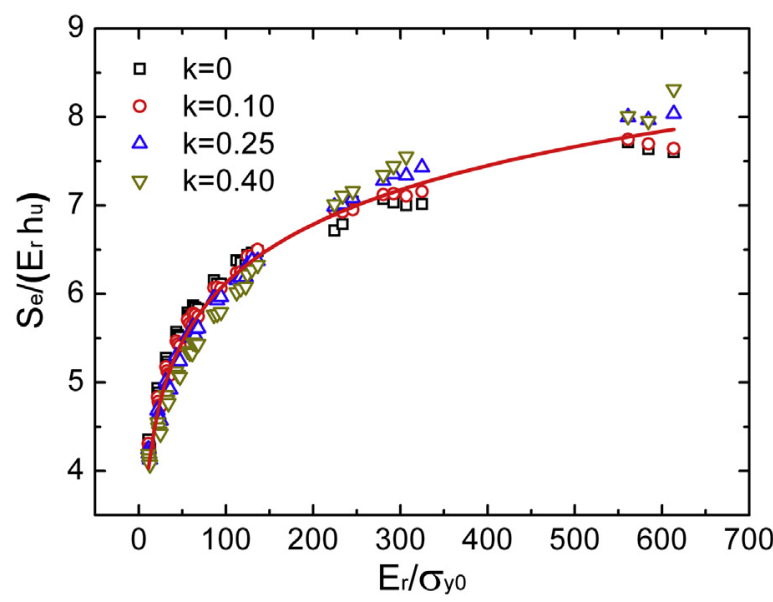

Fig. 6. Dimensionless function $\Pi_{2}$ obtained using 180 simulations. Solid curve is the best-fitting logarithmic curve to all data points.

\section{Experiments}

\subsection{Specimens}

Indentation tests and uniaxial tensile tests were performed on UPVC (Anheda Plastic Products Co., Ltd., Suzhou, China). For uniaxial tensile tests, the UPVC plates were processed into dumbbell-shaped specimens according to ISO 527-2. For indentation tests, the plates were processed into small specimens measuring $20 \mathrm{~mm} \times 20 \mathrm{~mm} \times 4 \mathrm{~mm}$. The glass transition temperature of UPVC is $87^{\circ} \mathrm{C}$. Hence, all specimens were annealed at $102{ }^{\circ} \mathrm{C}$ for $2.5 \mathrm{~h}$ in air to relieve the residual stress caused by mechanical processing.

\subsection{Indentation tests}

In order to extract the shear creep compliance, the yield strength under pure shear deformation and the coefficient of internal friction from a single $F$ - $h$ curve, the indentation data used in the present work are the same as used in our previous work [25]. Consequently, new indentation tests were not performed here. The details of the indentation tests in our previous work [25] were presented as follows.

The indentation tests were performed at the room temperature $\left(23^{\circ} \mathrm{C}\right)$ using a MTS Nano Indenter XP system (MTS Nano Instruments, Oak Ridge, TN) with a Berkovich indenter, which can be modeled as an equivalent cone with an included half-angle of $70.3^{\circ}$. An approximate step-holdunload loading profile was adopted. The load was increased quickly to the maximum $(13 \mathrm{mN})$ in $2 \mathrm{~s}$, held at the maximum load for $300 \mathrm{~s}$ and finally decreased linearly to zero in $50 \mathrm{~s}$. The indentation test was repeated 5 times.

\subsection{Uniaxial tensile tests}

A material testing system MTS 810 (MTS, Minneapolis, $\mathrm{MN}$ ) was used to perform the uniaxial tensile tests at the room temperature $\left(23^{\circ} \mathrm{C}\right)$. The tests were carried out in a displacement controlled manner, and the displacement increased at a constant extension rate. Four levels of extension rates, i.e. $10 \mathrm{~mm} / \mathrm{min}, 3 \mathrm{~mm} / \mathrm{min}, 1 \mathrm{~mm} / \mathrm{min}$ and $0.3 \mathrm{~mm} / \mathrm{min}$, were performed in the uniaxial tensile tests in order to probe the effect of strain rate on yield strength. The test of each extension rate was repeated 3 times.

\section{Results and discussion}

\subsection{Elastic modulus}

Fig. 7 shows the indentation $F$ - $h$ curves for UPVC. The values of contact stiffness, $S$, listed in Table 2 were obtained by first fitting a polynomial, $h=h_{\mathrm{f}}+a_{1} F^{1 / 2}+a_{2} F^{1 / 4}+a_{3} F^{1 / 8}$, to $45 \%$ of the unloading curves and then evaluating the derivative, $d F / d h$, at $h=h_{\mathrm{u}}$. The elastic contact stiffness, $S_{\mathrm{e}}$, was then calculated by Eq. (4). Replacing $S$ in Eqs. (1) and (3) with $S_{e}$, the reduced modulus was determined. The average reduced modulus of UPVC is $3.92 \mathrm{GPa}$, and the Poisson's ratio of UPVC usually takes a value of 0.4 . Consequently, the elastic modulus of UPVC was calculated by Eq. (2). As listed in Table 3, the average elastic modulus of UPVC determined by indentation test, $E_{\mathrm{IT}}=3.30 \mathrm{GPa}$, 


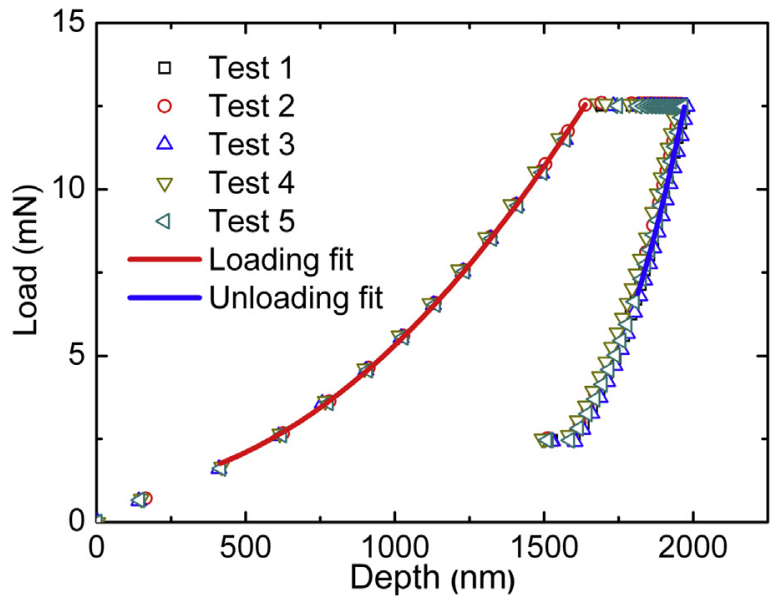

Fig. 7. Indentation load-depth curves of UPVC.

agrees well with that determined by uniaxial tensile test, $E=3.10 \mathrm{GPa}$, with a relative error of $6.45 \%$.

\subsection{Yield strength}

As shown in Fig. 7, the loading curvature, $C$, was obtained by fitting the loading curve (in the range from $400 \mathrm{~nm}$ to $1600 \mathrm{~nm}$ ) with $F=C h^{2}$. Then, using Eq. (23) and Eq. (24), the yield strength under the pure shear deformation, $\sigma_{\mathrm{y} 0}$, and the coefficient of internal friction, $k$, were determined (Table 2). The average values of $\sigma_{\mathrm{y} 0}$ and $k$ were $74.66 \mathrm{MPa}$ and 0.10 , respectively.

Fig. 8 shows the true stress-strain curves of uniaxial tensile tests at four levels of strain rate, and Fig. 9 reveals the effects of strain rate on the tensile yield strength. It was found that the tensile yield strength increases linearly with the logarithm of the strain rate. Fitting a logarithmic function, $\sigma_{\mathrm{yt}}=B \ln (\dot{\varepsilon})+D$, to the data points shown in Fig. 9, the coefficient, $B=2.58 \mathrm{MPa}$, was obtained.

For uniaxial tensile tests, the hydrostatic pressure, $P=-\sigma_{\mathrm{t}} / 3$, where $\sigma_{\mathrm{t}}$ is the true stress, and the effective stain rate, $\dot{\bar{\varepsilon}}=\dot{\varepsilon}$, where $\dot{\varepsilon}$ is the true strain rate. Thus, Eq. (8) can then be rewritten as

$\sigma_{y t}=\sigma_{y 0}-k \frac{\sigma_{y t}}{3}+B \ln (\dot{\varepsilon})$

where $\sigma_{\mathrm{yt}}$ is the tensile yield strength. Substituting the values of characteristic parameters $\left(\sigma_{\mathrm{y} 0}, k\right.$ and $\left.B\right)$ obtained

\section{Table 2}

Testing parameters and elastic-plastic parameters of UPVC determined by instrumented indentation.

\begin{tabular}{llllllll}
\hline Test no. $\begin{array}{l}S(\mathrm{mN} / \\
\mu \mathrm{m})\end{array}$ & $\begin{array}{l}S_{\mathrm{e}}(\mathrm{mN} / \\
\mu \mathrm{m})\end{array}$ & $C(\mathrm{GPa})$ & $E_{\mathrm{r}}(\mathrm{GPa})$ & $E(\mathrm{GPa})$ & $\sigma_{\mathrm{y} 0}(\mathrm{MPa})$ & $k$ \\
\hline Test 1 & 42.38 & 41.33 & 4.36 & 3.88 & 3.27 & 71.74 & 0.12 \\
Test 2 & 43.05 & 42.17 & 4.36 & 3.98 & 3.36 & 74.99 & 0.09 \\
Test 3 & 42.51 & 41.69 & 4.30 & 3.89 & 3.28 & 73.46 & 0.10 \\
Test 4 & 42.24 & 41.50 & 4.45 & 3.95 & 3.33 & 76.84 & 0.10 \\
Test 5 & 41.66 & 41.06 & 4.32 & 3.87 & 3.26 & 76.25 & 0.08 \\
Average & 42.37 & 41.55 & 4.36 & 3.92 & 3.30 & 74.66 & 0.10 \\
\hline
\end{tabular}

Table 3

Comparison between indentation and uniaxial tensile results of UPVC. The results listed below are average values of elastic modulus and tensile yield strength at different strain rates. The subscript IT indicates indentation test.

\begin{tabular}{|c|c|c|c|c|c|c|}
\hline \multicolumn{3}{|c|}{ Elastic modulus } & \multicolumn{4}{|c|}{ Tensile yield strength } \\
\hline$E(\mathrm{GPa})$ & $E_{\mathrm{IT}}(\mathrm{GPa})$ & Rel. Err. & $\dot{\varepsilon}\left(\mathrm{s}^{-1}\right)$ & $\sigma_{\mathrm{yt}}(\mathrm{MPa})$ & $\sigma_{\mathrm{yt} \_\mathrm{IT}}(\mathrm{MPa})$ & Rel. Err. \\
\hline \multirow[t]{4}{*}{3.10} & 3.30 & $6.45 \%$ & $1.40 \times 10^{-3}$ & 60.53 & 55.91 & $-7.64 \%$ \\
\hline & & & $4.20 \times 10^{-4}$ & 56.87 & 52.91 & $-6.96 \%$ \\
\hline & & & $1.40 \times 10^{-4}$ & 54.47 & 50.17 & $-7.90 \%$ \\
\hline & & & $4.30 \times 10^{-5}$ & 51.91 & 47.22 & $-9.03 \%$ \\
\hline
\end{tabular}

above and the true strain rate into Eq. (25), the tensile yield strength at corresponding strain rate was determined. As listed in Table 3, the tensile yield strengths estimated by indentation tests, $\sigma_{\text {yt_IT, }}$ were compared with those determined by uniaxial tensile tests, $\sigma_{\text {yt }}$. The maximum relative error is $-9.03 \%$, which indicates that the method proposed in the present work can relatively accurately determine the plastic properties of glassy polymers.

\subsection{Shear relaxation modulus}

Based on linear viscoelastic theory, the relationships between shear creep compliance, $J(t)$, and shear relaxation modulus, $G(t)$, can be expressed as

$\int_{0}^{t} J(t-\tau) \frac{d G(\tau)}{d \tau} d \tau=1$

or

$\int_{0}^{t} G(t-\tau) \frac{d J(\tau)}{d \tau} d \tau=1$

Theoretically, the shear creep compliance can be converted to shear relaxation modulus as long as the shear creep compliance is known, and vice versa [35,36]. The shear creep compliance and shear relaxation modulus can be represented by Prony series as

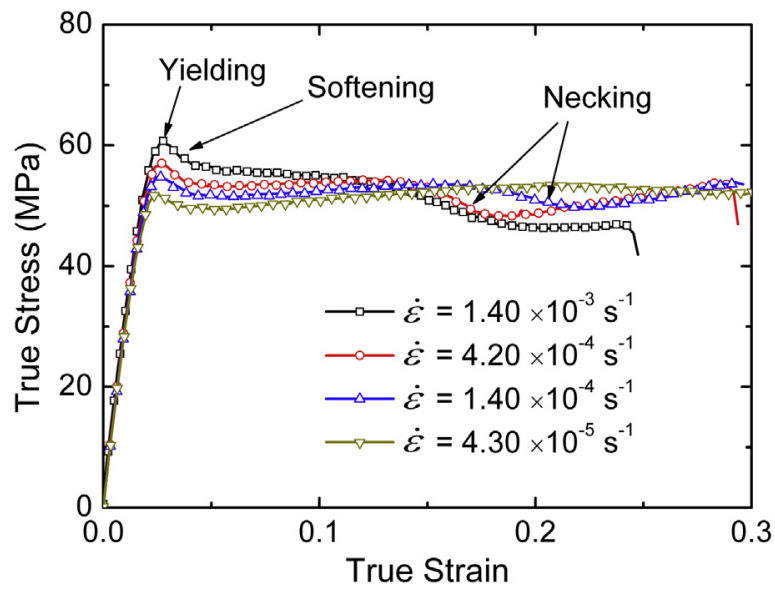

Fig. 8. True stress-strain curves of UPVC obtained by uniaxial tensile tests at four strain rates. 


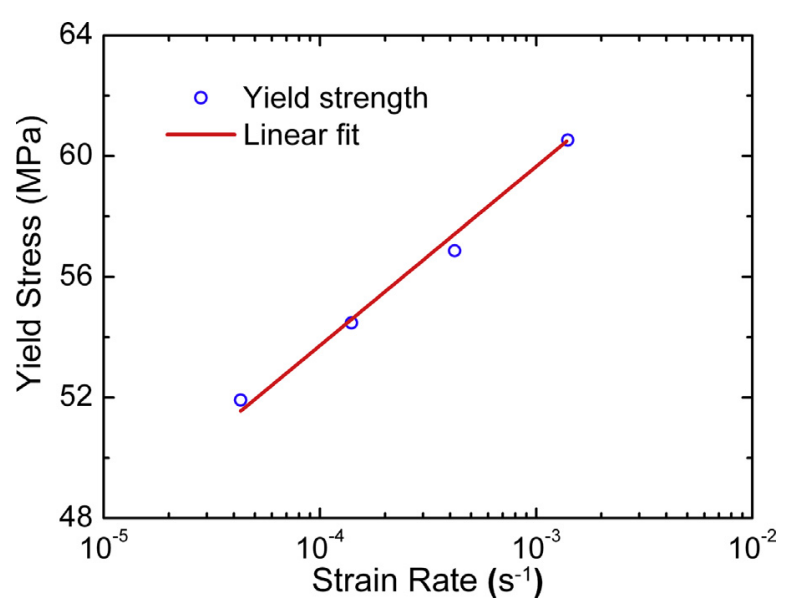

Fig. 9. Effect of strain rate on tensile yield strength of UPVC.

$J(t)=J_{\infty}-\sum_{i=1}^{N} J_{i} e^{-t / \tau_{i}}$

and

$G(t)=G_{0}+\sum_{j=1}^{M} G_{j}\left(e^{-t / \xi_{j}}-1\right)$

where $J_{\infty}$ is the long-term shear creep compliance; $J_{i}$ and $\tau_{i}$ are retardation strengths and retardation times, respectively; $G_{0}$ is the instantaneous shear modulus; $G_{j}$ and $\xi_{j}$ are relaxation strengths and relaxation times, respectively; $N$ and $M$ are positive integers. Substituting Eq. (28) and Eq. (29) into Eq. (26), and assuming the retardation time equals the relaxation time for the same order, i.e. $\tau_{i}=\xi_{i}$, we get

$$
\begin{aligned}
& \sum_{j=1}^{M}\left[J_{\infty}\left(e^{-t / \tau_{j}}-1\right)+\sum_{i=1, i \neq j}^{N} \frac{\tau_{i}}{\tau_{i}-\tau_{j}} J_{i}\left(e^{-t / \tau_{i}}-e^{-t / \tau_{j}}\right)+\frac{t}{\tau_{j}} J_{j} e^{-t / \tau_{j}}\right] G_{j} \\
& =1-G_{0}\left(J_{\infty}-\sum_{i=1}^{N} J_{i} e^{-t / \tau_{i}}\right)
\end{aligned}
$$

When a set of constants, either $\left\{J_{\infty}, J_{i}\right.$ and $\left.\tau_{i}(i=1, \cdots, N)\right\}$ or $\left\{G_{0}, G_{j}\right.$ and $\left.\xi_{j}(j=1, \cdots, M)\right\}$, are known, the other set of constants can be determined by fitting Eq. (30) to a series of time points $\left(t=t_{1}, t_{2}, t_{3}, \cdots\right)$. Then, substituting the corresponding set of constants back into Eq. (28) or Eq. (29), the shear creep compliance or shear relaxation modulus can be obtained.

The shear creep compliance of UPVC has been determined via indentation tests in our previous work [25]. If the order of shear creep compliance takes a value of 3 , it can be expressed by Prony series as

$$
\begin{aligned}
J(t)= & 1.240-0.103 e^{-t / 10.913}-0.085 e^{-t / 47.604} \\
& -0.120 e^{-t / 304.944}
\end{aligned}
$$

Consequently, the creep constants $\left\{J_{\infty}, J_{i}\right.$ and $\tau_{i}(i=1,2$, $3)\}$ in Eq. (30) were known. If the order of shear relaxation modulus also takes a value of 3, the fitting into Eq. (30) allows the determination of a set of relaxation constants
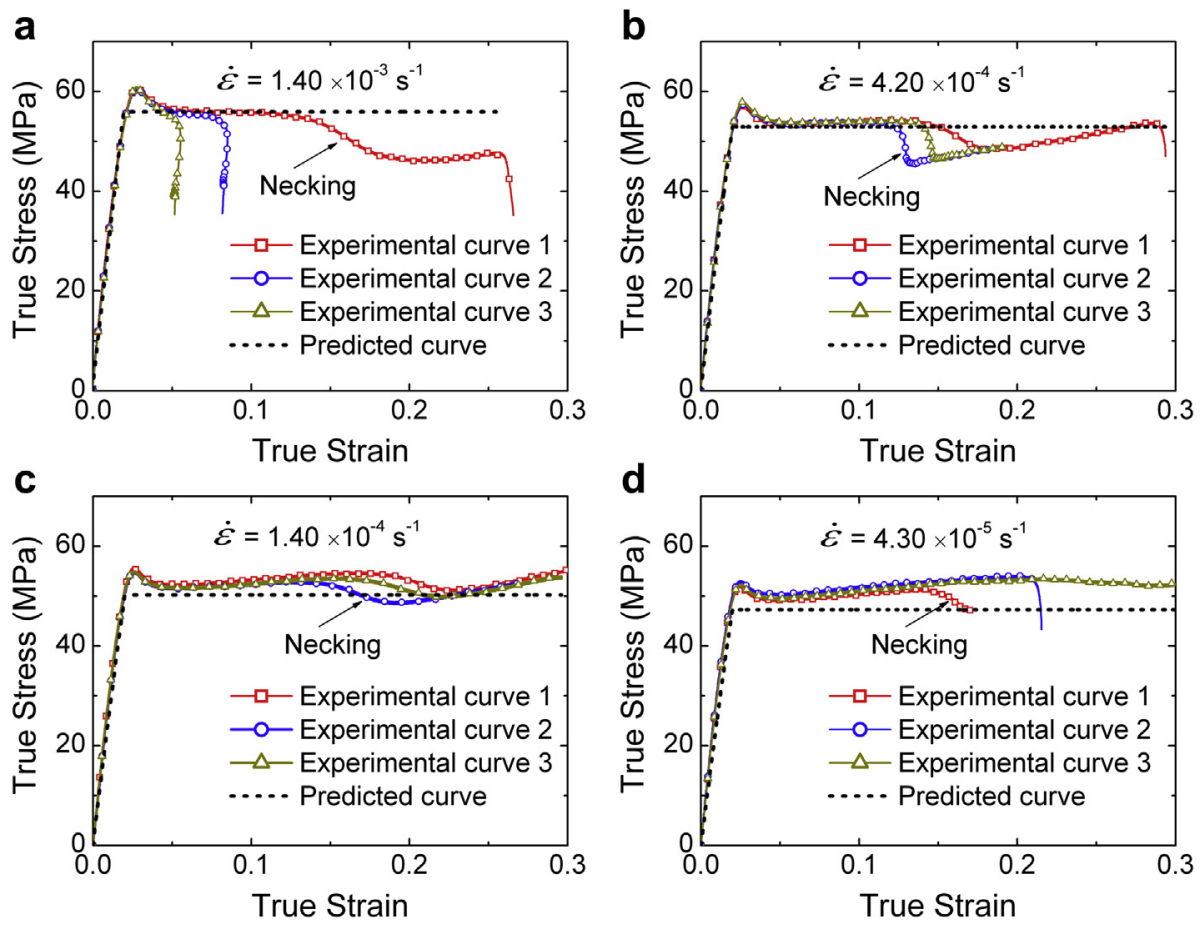

Fig. 10. Comparison between predicted and experimental true stress-strain curves at (a) $\dot{\varepsilon}=1.40 \times 10^{-3} \mathrm{~s}^{-1},(\mathrm{~b}) \dot{\varepsilon}=4.20 \times 10^{-4} \mathrm{~s}^{-1}$, (c) $\dot{\varepsilon}=1.40 \times 10^{-4} \mathrm{~s}^{-1}$ and $(\mathrm{d}) \dot{\varepsilon}=4.30 \times 10^{-5} \mathrm{~s}^{-1}$. 
$\left\{G_{0}, G_{j}\right.$ and $\left.\xi_{j}(j=1,2,3)\right\}$. Substituting these relaxation constants back into Eq. (29), the shear relaxation modulus in the form of Prony series was determined

$$
\begin{aligned}
G(t)= & 1.073+0.122\left(e^{-t / 10.913}-1\right)+0.064\left(e^{-t / 47.604}-1\right) \\
& +0.086\left(e^{-t / 304.944}-1\right)
\end{aligned}
$$

\subsection{Prediction of uniaxial tensile curve}

Since the viscoelastic-plastic properties of UPVC have been determined by indentation tests, the true stressstrain curves of UPVC can be predicted. For each uniaxial tensile test, the true strain rate, $\dot{\varepsilon}$, is a constant. Thus, the variation of the true stain can be evaluated by $\varepsilon(t)=\dot{\varepsilon} t$. Based on the linear viscoelastic integral equations, the true stress before yielding is calculated by

$\sigma(t)=2(1+\nu) \dot{\varepsilon} \int_{0}^{t} G(t-\tau) d \tau$

where $G(t)$ was already determined [Eq. (32)]. When the true stress reaches the yield strength (Table 3 ), the specimens yield. According to the linear viscoelastic-perfectly plastic description, the true stress remains constant after yielding.

The predicted true stress-strain curves were compared with the experimental curves in Fig. 10. It is clear that the predicted curves are in good agreement with the experimental curves before necking with a maximum difference of $-12.39 \%$. This demonstrates that the viscoelastic-plastic properties determined by our methods can be used to predict the uniaxial tensile curves. In other words, the method for determining shear creep compliance in our previous work [25] and the method for extracting the yield strength under pure shear deformation and the coefficient of internal friction in the present work are reliable methods for characterization of the viscoelastic-plastic properties of glassy polymers.

\section{Conclusions}

Based on the assumption that the behavior of glassy polymers can be approximately characterized by a linear viscoelastic-perfectly plastic description, we established a method for determining the yield strength under pure shear deformation, $\sigma_{\mathrm{y} 0}$, and the coefficient of internal friction, $k$, by instrumented indentation. Together with the method for determining shear creep compliance proposed in our previous work [25], the viscoelastic-plastic properties of glassy polymers can be extracted from a single indentation $F$ - $h$ curve.

The tensile yield strengths of UPVC estimated by indentation tests agree well with those determined by uniaxial tensile tests. The true stress-strain curves of UPVC predicted using the material parameters determined by indentation tests are in good agreement with those obtained by tensile tests before necking. This demonstrates that the method for determining shear creep compliance in our previous work [25] and the method for extracting $\sigma_{\mathrm{y} 0}$ and $k$ in the present work are reliable methods for characterization of the viscoelastic-plastic properties of glassy polymers.

\section{Acknowledgements}

The authors gratefully acknowledge the support from National Natural Science Foundation of China (Grant Nos. $11025212,11272318,11302231$, and 11372323).

\section{References}

[1] W.C. Oliver, G.M. Pharr, An improved technique for determining hardness and elastic modulus using load and displacement sensing indentation experiments, J. Mater. Res. 7 (1992) 1564-1583.

[2] M. Dao, N. Chollacoop, K.J.V. Vliet, T.A. Venkatesh, S. Suresh, Computational modeling of the forward and reverse problems in instrumented sharp indentation, Acta Mater. 49 (2001) 3899-3918.

[3] J.L. Bucaille, S. Stauss, E. Felder, J. Michler, Determination of plastic properties of metals by instrumented indentation using different sharp indenters, Acta Mater. 51 (2003) 1663-1678.

[4] Y.P. Cao, J. Lu, A new method to extract the plastic properties of metal materials from an instrumented spherical indentation loading curve, Acta Mater. 52 (2004) 4023-4032.

[5] J. Luo, J. Lin, A study on the determination of plastic properties of metals by instrumented indentation using two sharp indenters, Int. J. Solids Struct. 44 (2007) 5803-5817.

[6] P. Jiang, T.H. Zhang, Y.H. Feng, R. Yang, N.G. Liang, Determination of plastic properties by instrumented spherical indentation: expanding cavity model and similarity solution approach, J. Mater. Res. 24 (2009) 1045-1053.

[7] I.N. Sneddon, The relation between load and penetration in the axisymmetric Boussinesq problem for a punch of arbitrary profile, Int. J. Eng. Sci. 3 (1965) 47-57.

[8] J.C. Hay, A. Bolshakov, G.M. Pharr, A critical examination of the fundamental relations used in the analysis of nanoindentation data, J. Mater. Res. 14 (1999) 2296-2305.

[9] J.L. Hay, G.M. Pharr, Instrumented indentation testing, in: H. Kuhn, D. Medlin (Eds.), Mechanical Testing and Evaluation, ASM Handbook, Vol. 8, ASM International, Materials Park, Ohio, 2000, pp. 232 243.

[10] G. Feng, A.H.W. Ngan, Effects of creep and thermal drift on modulus measurement using depth-sensing indentation, J. Mater. Res. 17 (2002) 660-668.

[11] A.H.W. Ngan, B. Tang, Viscoelastic effects during unloading in depth-sensing indentation, J. Mater. Res. 17 (2002) 2604-2610.

[12] B. Tang, A.H.W. Ngan, Accurate measurement of tip-sample contact size during nanoindentation of viscoelastic materials, J. Mater. Res. 18 (2003) 1141-1148.

[13] Y.T. Cheng, C.M. Cheng, Relationships between initial unloading slope, contact depth, and mechanical properties for spherical indentation in linear viscoelastic solids, Mater. Sci. Eng. A 409 (2005) 93-99.

[14] Y.T. Cheng, W. Ni, C.M. Cheng, Determining the instantaneous modulus of viscoelastic solids using instrumented indentation measurements, J. Mater. Res. 20 (2005) 3061-3071.

[15] E.H. Lee, J.R.M. Radok, The contact problem for viscoelastic bodies, J. Appl. Mech. 27 (1960) 438-444.

[16] S.C. Hunter, The hertz problem for a rigid spherical indenter and a viscoelastic half-space, J. Mech. Phys. Solids 8 (1960) 219-234.

[17] G.A.C. Graham, The contact problem in the linear theory of viscoelasticity, Int. J. Eng. Sci. 3 (1965) 27-46.

[18] T.C.T. Ting, Contact stresses between a rigid indenter and a viscoelastic half-space, J. Appl. Mech. 33 (1966) 845-854.

[19] H. Lu, B. Wang, J. Ma, G. Huang, H. Viswanathan, Measurement of creep compliance of solid polymers by nanoindentation, Mech. Time-Depend. Mat. 7 (2003) 189-207.

[20] M.R. VanLandingham, N.K. Chang, P.L. Drzal, C.C. White, S.H. Chang, Viscoelastic characterization of polymers using instrumented indentation. I. Quasi-static testing, J. Polym. Sci. Part B: Polym. Phys. 43 (2005) 1794-1811.

[21] M.L. Oyen, Analytical techniques for indentation of viscoelastic materials, Philos. Mag. 86 (2006) 5625-5641.

[22] C.A. Tweedie, K.J. Van Vliet, Contact creep compliance of viscoelastic materials via nanoindentation, J. Mater. Res. 21 (2006) 1576-1589.

[23] J. Menčík, L. Beneš, Determination of viscoelastic properties by nanoindentation, J. Optoelectron. Adv. M. 10 (2008) 3288-3291. 
[24] Y.T. Cheng, F.Q. Yang, Obtaining shear relaxation modulus and creep compliance of linear viscoelastic materials from instrumented indentation using axisymmetric indenters of power-law profiles, J. Mater. Res. 24 (2009) 3013-3017.

[25] G.J. Peng, T.H. Zhang, Y.H. Feng, Y. Huan, Determination of shear creep compliance of linear viscoelastic-plastic solids by instrumented indentation, Polym. Test. 31 (2012) 1038-1044.

[26] G.J. Peng, T.H. Zhang, Y.H. Feng, R. Yang, Determination of shear creep compliance of linear viscoelastic solids by instrumented indentation when the contact area has a single maximum, J. Mater. Res. 27 (2012) 1565-1572.

[27] P.B. Bowden, J.A. Jukes, The plastic flow of isotropic polymers, J. Mater. Sci. 7 (1972) 52-63.

[28] R.A. Duckett, S. Rabinowi, I.M. Ward, The strain-rate, temperature and pressure dependence of yield of isotropic poly(methylmethacrylate) and poly(ethylene terephthalate), J. Mater. Sci. 5 (1970) 909-915.

[29] A.A. Silano, K.D. Pae, J.A. Sauer, Effects of hydrostatic pressure on shear deformation of polymers, J. Appl. Phys. 48 (1977) 4076.
[30] A.W. Christiansen, E. Baer, S.V. Radcliffe, The mechanical behaviour of polymers under high pressure, Philos. Mag. 24 (1971) 451-467.

[31] H. Ghoneim, Y. Chen, A viscoelastic-viscoplastic constitutive equation and its finite element implementation, Comput. Struct. 17 (1983) 499-509.

[32] R. Quinson, J. Perez, M. Rink, Yield criteria for amorphous glassy polymers, J. Mater. Sci. 32 (1997) 1371-1379.

[33] C.Y. Zhang, Y.W. Zhang, K.Y. Zeng, L. Shen, Characterization of mechanical properties of polymers by nanoindentation tests, Philos. Mag. 86 (2006) 4487-4506.

[34] L. Brand, The Pi theorem of dimensional analysis, Arch. Ration. Mech. 1 (1957) 35-45.

[35] S.W. Park, R.A. Schapery, Methods of interconversion between linear viscoelastic material functions. Part I - a numerical method based on Prony series, Int. J. Solids Struct. 36 (1999) 1653-1675.

[36] J. Sorvari, M. Malinen, Numerical interconversion between linear viscoelastic material functions with regularization, Int. J. Solids Struct. 44 (2007) 1291-1303. 